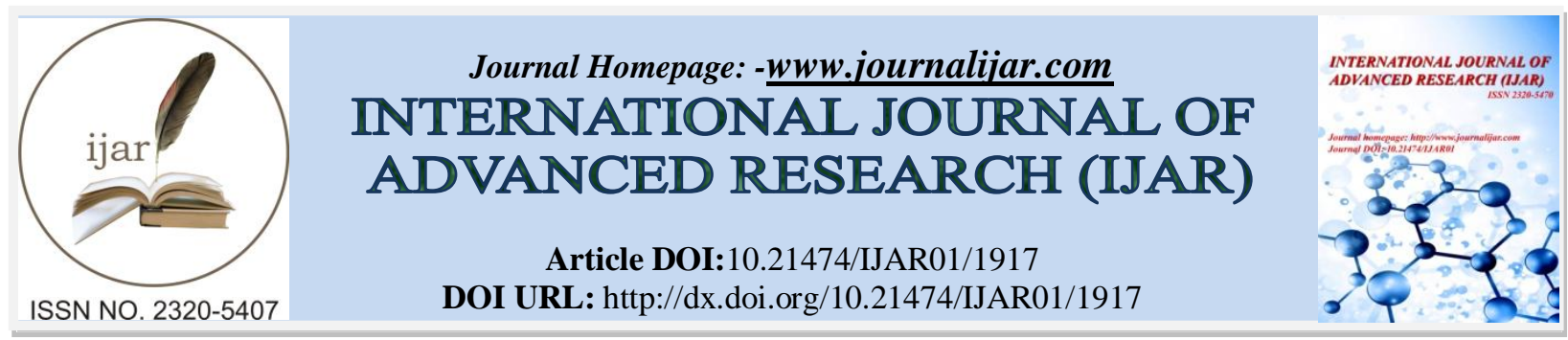

RESEARCH ARTICLE

\title{
PREGNANCY COMPLICATED WITH HEREDITARY SPHEROCYTOSIS - LESSONS LEARNT FROM A CASE AND REVIEW OF LITERATURE.
}

Parul Verma ${ }^{1}$ and Shashikant R. Kulkarni ${ }^{2}$.

1. Junior Resident, Department of Obstetrics and Gynaecology, Dr. D.Y. Patil Medical College,Kolhapur, Maharashtra-416003, India.

2. Professor and Head, Department of Obstetrics and Gynaecology, Dr. D.Y. Patil Medical College, Kolhapur, Maharashtra-416003, India.

\section{Manuscript Info}

(..........................

Manuscript History

Received: 12 August 2016

Final Accepted: 22 September 2016

Published: October 2016

Key words:-

Hereditary spherocytosis, Pregnancy,

Splenectomy

\section{Abstract}

Hereditary spherocytosis (HS) also known as Minkowski-Chauffard syndrome is a genetically determined disorder of the red blood cell membrane cytoskeleton complex causing Hemolytic anemia. There is a wide spectrum of clinical presentation ranging from mild anemia to severe anemia with splenomegaly and jaundice. Increased Mean Corpuscular Hemoglobin Concentration (MCHC), spherocytes in peripheral blood smear and increased osmotic fragility favour the diagnosis of HS.No causal treatment is yet available for this disease. Mild disease warrants no treatment. Folic acid, supportive treatment and regular annual check up usually suffice for moderate disease while frequent hematological supervision and splenectomy is prudent for severe disease.Pregnancy complicates the management of HS and very limited data is available in the literature regarding this.

Case Presentation:-Authors hereby report a case of 24-year-old 36weeksgestational age primigravida withintrauterine fetal demise. Workup revealed patient to be suffering from Hereditary Spherocytosis that resulted in hemolysis ultimately causing fetal demise. Pregnancy was terminated and patient was subsequently referred to the Department of Surgery for splenectomy.

Conclusion:- Hereditary Spherocytosis, one of the commonest congenital hemolytic anemia may get decompensated during pregnancy. Due to paucity of data in literature regarding management of HS during pregnancy, it is hard to formulate guidelines for indications of splenectomy during pregnancy. However, authors recommend Splenectomy preferably in second trimester in any pregnant women with HS who is experiencing hemolysis or having symptoms, signs and complications thereof. Obstetricians should exercise high index of suspicion to diagnose this not so uncommon entity as early as pregnancy is diagnosed to yield better outcomes.

Copy Right, IJAR, 2016,. All rights reserved. 


\section{Introduction:-}

Hereditary spherocytosis (HS) is a genetically determined disorder of the red blood cell membrane cytoskeleton complex causing Hemolytic anemia. The estimated frequency of HS varies from 1:2000 to 1:5000. ${ }^{1-3}$ Minkowski and Chauffard in the late $19^{\text {th }}$ century reported families whose peripheral blood revealed numerous spherocytes and have been thus duly credited with the discovery of this not so uncommon disorder. ${ }^{4,5}$ The red cells were abnormally susceptible to lysis in hypotonic media as revealed by in vitro studies, thereby establishing osmotic fragility test virtually diagnostic of HS.

There is a wide spectrum of clinical presentation ranging from mild anemia to severe anemia with splenomegaly and jaundice. Increased Mean Corpuscular Hemoglobin Concentration (MCHC), spherocytes in peripheral blood smear and increased osmotic fragility favour the diagnosis of HS.Sodium dodecyl sulphate polyacrylamide gel electrophoresis (SDS-PAGE) is usually confirmative. ${ }^{1,2}$

No causal treatment is yet available for this disease. Mild disease warrants no treatment. Folic acid, supportive treatment and regular annual check up usually suffice for moderate disease while frequent hematological supervision and splenectomy is prudent for severe disease. ${ }^{6}$ It is a well known fact that intercurrent conditions like, pregnancy and infections may cause decompensation in a previously well compensated disease but unfortunately very limited data is available regarding the course of HS during pregnancy with very few cases reported in the literature till date. ${ }^{7}$ Authors hereby report a case of pregnancy complicated with intrauterine fetal demise who was later diagnosed to be suffering from HS.

\section{Case report:-}

A 24-year-old 36-weeks gestational age primigravida presented in the emergency department with the complaints of decreased fetal movement since morning. She was married for 11 months and had normal menstrual cycles.Past history was insignificant except for one unit Packed red blood cell transfusion at the age of 12 years. Examination revealed pallor and icterus. Abdominal examination showed gravid uterus with fundal height corresponding to 32-34 weeks gestational age with longitudinal lie and cephalic presentation. Head was found to be floating. No fetal movements were felt during examination. Fetal Heart sounds could not be auscultated. Patient was relaxed and there was no evidence of uterine contractions. Per speculum examination showed Cervix and Vagina to be healthy. There was no evidence of per vaginal discharge or bleeding. Per vaginum examination revealed os to be closed, posteriorly placed and was uneffaced.

Obstetric Ultrasonography examination revealed single intrauterine fetus of 38 weeks 0 day in cephalic presentation with absent fetal cardiac activity and fetal movements suggestive of fetal demise. Complete blood count showed anemia with $9.9 \mathrm{~g}$ Hemoglobin/dL, leukocytosis with 15600 total leucocyte counts/ cubic mm. Mean Corpuscular Volume (MCV) was 95.9 fL,Mean Corpuscular Hemoglobin (MCH)was 33.9 pg and Mean Corpuscular Hemoglobin Concentration (MCHC)was $35.4 \mathrm{~g} / \mathrm{dL}$. Peripheral blood smear showed increased number of spherocytes (++) with few polychromatophils. Spherocytes were uniform in size and density without any central pallor. Platelets were adequate in number with 2.81 lacs/ cubic mm. Reticulocytosis was present with $8.0 \%$ reticulocytes. Liver function tests revealed indirect hyperbilirubinemia with $5.7 \mathrm{mg}$ total serum bilirubin/dL and 0.9 $\mathrm{mg}$ direct serum bilirubin/dL. Liver enzymes were normal.Renal Function Tests were normal. Serum Lactate dehydrogenase was found to be raised with values of 566.9 Units/L. Iron studies, Glucose-6-Phosphate Dehydrogenase (G6PD) assay and Hemoglobin electrophoresis were normal. Patient tested negative for antinuclear antibody (ANA), Indirect and Direct Coomb's test. The above findings were strongly suggestive of Hemolytic anemia most probably as a result of Hereditary Spherocytosis. Subsequently Osmotic fragiltiy test was done which concluded Initial lysis (minimal resistance) in $0.745 \% \mathrm{NaCl}$, Complete lysis (maximum resistance) in $0.33 \% \mathrm{NaCl}$ and Mean corpuscular fragilty in $0.515 \% \mathrm{NaCl}$, thereby confirming the diagnosis of Hereditary Spherocytosis.

While enquiring about the old records, patient showed six months old reports. Complete blood counts showed anemia with $10.6 \mathrm{~g} \mathrm{Hb} / \mathrm{dL}$, leucocytosis with $12130 \mathrm{TLC} / \mathrm{cumm}$ and 2.64 lacs Platelets / cumm. MCV was 89.6 $\mathrm{fL}, \mathrm{MCH}$ was $30.0 \mathrm{pg}$ and $\mathrm{MCHC}$ was $33.5 \%$.Indirect hyperbilirubinemia with $3.2 \mathrm{mg} / \mathrm{dL}$ total serum bilirubin and $1.6 \mathrm{mg} / \mathrm{dL}$ direct fraction was seen in LFT. USG abdomen done at the periphery showed minimal hepatomegaly with multiple calculi in Gall bladder with moderate splenomegaly and a single live early intrauterine pregnancy of gestational age 12 weeks 5 days. 
A diagnosis of 36 weeks pregnancy with Hereditary Spherocytosis with Intrauterine fetal demise was made and was planned to terminate the pregnancy.

\section{Treatment and outcome:-}

Induction of labour was started with Dinoprostone (Prostaglandin E2) gel followed by insertion of intracervical catheter. Labour was augmented by Oxytocin infusion and dead fetus was delivered. Postpartum period was uneventful. Patient was prescribed Folic acid $5 \mathrm{mg}$ per day and thereafter was referred to the Department of Surgery for Splenectomy.

Table1:-Classification of Hereditary Spherocytosis.

\begin{tabular}{|l|l|l|l|l|}
\hline CLASSIFICATION & Trait & Mild & Moderate & Severe \\
\hline Hemoglobin $(\mathrm{g} / \mathrm{dL})$ & Normal & $11-15$ & $8-12$ & $6-8$ \\
\hline Reticulocyte count $(\%)$ & Normal $(<3 \%)$ & $3-6$ & $>6$ & $>10$ \\
\hline Bilirubin $(\mu \mathrm{mol} / \mathrm{L})$ & $<17$ & $17-34$ & $>34$ & $>51$ \\
\hline $\begin{array}{l}\text { Spectrin per erythrocyte }(\% \text { of } \\
\text { normal) }\end{array}$ & 100 & $80-100$ & $50-80$ & $40-60$ \\
\hline
\end{tabular}

\section{Discussion:-}

Hereditary spherocytosis (HS) is one of the commonest congenital hemolytic anemia caused by genetic mutations in genes encoding red blood cell (RBC) membrane proteins. Various membrane proteins can be affected in HS like spectrin, ankyrin, band 3, and protein 4.2 but spectrin deficiency is reported to be the commonest culprit resulting in HS. Autosomal dominant pattern of inheritance is the most frequent mode of transmission of this genetically heterogeneous disease but few cases of autosomal recessive inheritance have also been reported. The estimated prevalence as reported in various systematic reviews range from 1 in 2000 to 1 in 5000 people. $^{1-3}$

Clinically, there is a wide spectrum of presentation ranging from asymptomatic mild anemia to fulminant hemolytic anemia with pallor, jaundice and splenomegaly requiring aggressive treatment. Gallstones of pigment type caused by increased unconjugated bilirubin may be the first presentation of this disorder. Family history is variably present with patients reporting history of splenectomy and cholecystectomy in family member. Environmental triggers, like, fatigue, infections, emotional distress and pregnancy have been reported to unmask the previously compensated disease. $^{7}$

The HS is characterized by mild to moderate anemia with increased mean corpuscular hemoglobin concentration (MCHC). Increased MCHC due to cellular dehydration is a hallmark of HS. Reticulocytosis is invariablypresent signifying increased cell production. Peripheral blood smear revealspherocytes, which in contrast to spherocytes seen in immune hemolytic anemia and thermal injury are quite uniform in size and density (Fig. - 1). Unconjugated hyperbilirubinemia is frequently observed due to hemolysis. Incubated osmotic fragility test is the most sensitive test for HS (Fig.-2). ${ }^{8}$ Other tests infrequently used are Acidified glycerol lysis time (AGLT), Flow cytometry (Eosin-5maleimide binding), Osmotic gradient ektacytometry and membrane analysis by SDS-PAGE. ${ }^{9}$ 


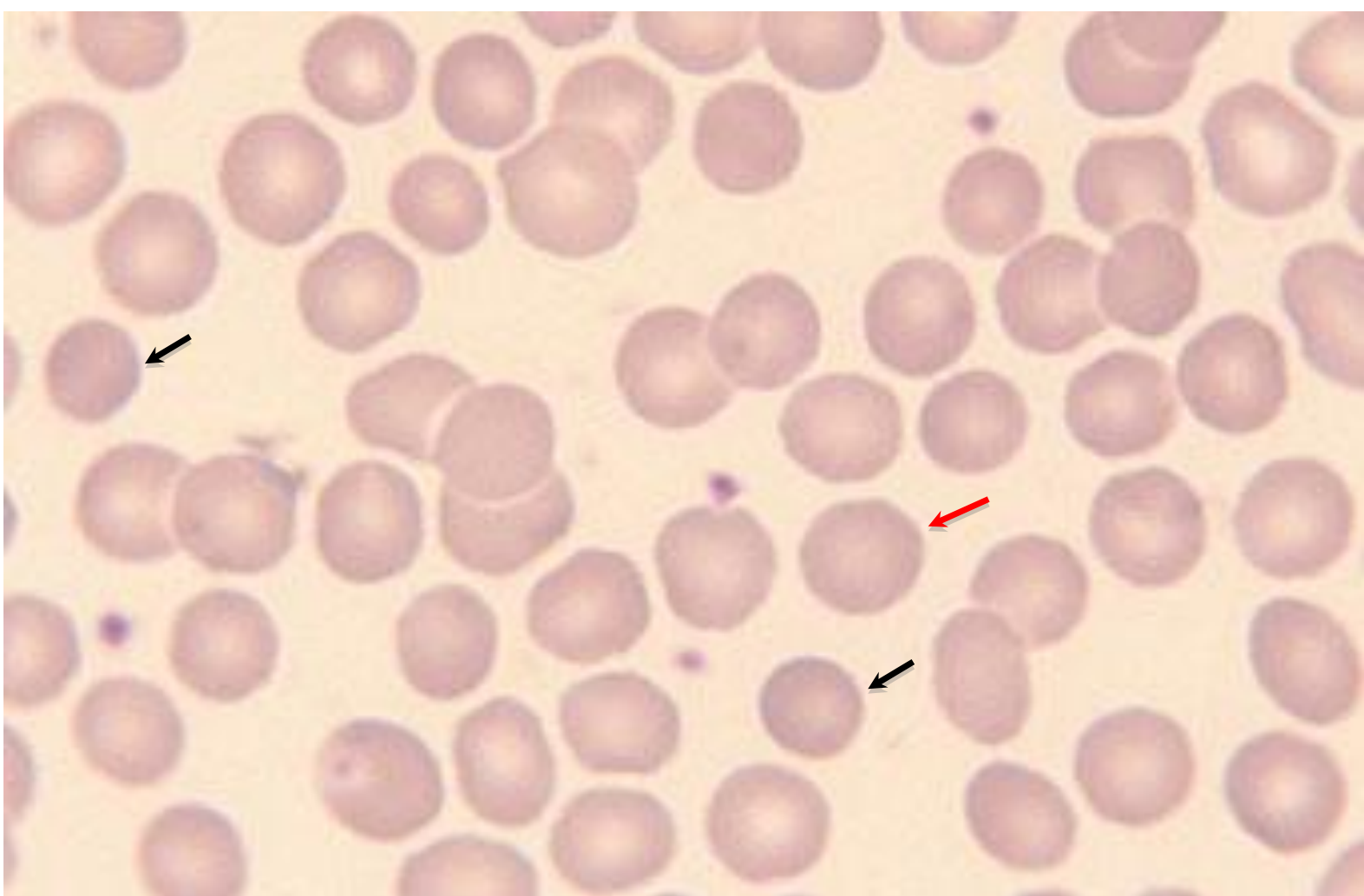

Fig.1:- Peripheral smear shows increased number of spherocytes (black arrow) and few polychromatophils (red arrow). Spherocytes are uniform in size and density without any central pallor.

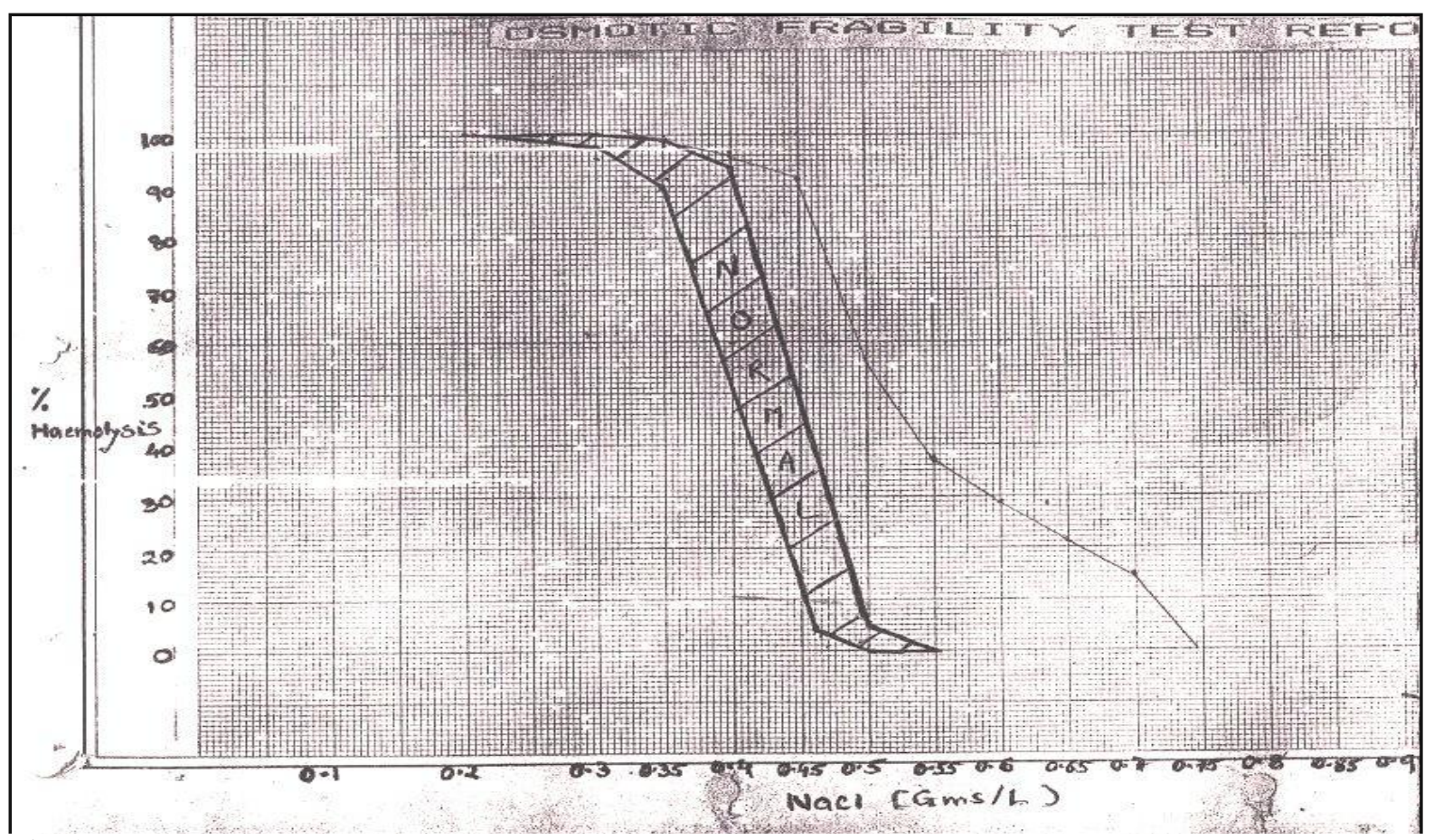

Fig. 2:- Osmotic Fragility Curve of the patient. The whole curve is shifted to the right. Please note the tailing of fragile erythrocytes, which are lysed at higher concentration of saline (0.7-0.8\%). Normally no lysis occurs at these concentrations. 
This disorder despite being discovered almost 2 centuries back, unfortunately, there is still no causative treatment to correct the basic disorder. Decision to offer treatment to the patients suffering from HS is based on the severity of clinical symptoms and complications (Table-1). ${ }^{10}$ Folate supplements have been routinely prescribed to the patients suffering from chronic hemolysis and folate therapy is recommended in moderate and severe HS but not in mild HS in the dose of $5 \mathrm{mg}$ per day.

Splenectomy has long been the standard treatment for patients with clinically severe HS and is considered in those with moderate HS. It should not be performed in mild HS. ${ }^{1,2,6}$ Splenectomy is a double edged sword, at one end it eliminates the sign and symptoms of hemolysis and at the other end it makes individual susceptible for life long risk of infections with capsulated organism, especially, Pneumococci with a mortality of $0.1-0.4 \%$. ${ }^{11,12}$ However, this risk has been reduced by vaccination against pneumococcus, meningococcus and Hemophilus influenzae coupled with prophylactic antibiotics. It has been recommended to vaccinate patients undergoing splenectomy two weeks prior to surgery. ${ }^{1-3,13}$ Recently, a largest single center study done by Thomas Pincez etal reported safety and success of subtotal splenectomy over total splenectomy in children. ${ }^{14}$ Concurrent cholecystectomy is advocated if the patient has symptoms of gall stone disease. ${ }^{1,2,12}$

Pregnancy complicates the management of hereditary spherocytosis. Traditionally, many authors have held the view of avoiding splenectomy during pregnancy due to fear of perioperative morbidity associated with the surgery to both mother and fetus. There is paucity of data in literature in this regard but the studies although few have been contrary to the long held concept of avoiding splenectomy during pregnancy.Second trimester is usually preferred for splenectomy, although it can be performed at any gestational age. Less invasive laparoscopic splenectomy is routinely preferred due to its obvious advantages over open splenectomy. ${ }^{3}$

Moore etal (1976) have reported two cases of pregnant women in the second trimester with HS who underwent successfulsplenectomy and reviewed 5 such cases in literature. ${ }^{15}$ Ho-Yen DO (1984) described a family with HS in which 3 sisters had hemolytic episodes during their 6 pregnancies but none was treated with splenectomy and each pregnancy resulted in birth of mature and live infant. ${ }^{16}$ Maberry etal (1992) reported 23 women with HS during their 50 pregnancies, none requiring splenectomy during pregnancy. Maternal complications were infrequent and perinatal outcomes were excellent. ${ }^{17}$ Pajor etal (1993) have reported 8 patients with HS whohad 19 pregnancies in total, 10 before splenectomy and 9 after splenectomy. Allwere managed conservatively and was found that fetal outcome was more favourable after splenectomy than before splenectomy. ${ }^{18}$ Brabec V etal (1999) studied the influence of pregnancy on the course of HS in 21 women during their 44 pregnancies and found that pregnancy caused no problems in the majority, even when complications developed they were not serious. Anemia deteriorated in one-third pregnancies in non-splenectomized women due to increased hemolysis, while complaints were minimal in splenectomized patients. ${ }^{19}$ Only two instances of successful laparoscopic splenectomy in a pregnant woman suffering from HS have been reported in literature yet. ${ }^{3,20}$

The case presented by the authors seems to have been undergoing hemolysis since last six months as evident by anemia and unconjugated hyperbilirubinemia in routine hematological reports and also there was evidence of hepatosplenomegaly in Ultrasonography study of abdomen but unfortunately it could not be recognized at the periphery. It was sad that patient presented to the authors when fetal demise already occurred. Had she been previously worked up and diagnosed as a case of HS and underwent splenectomy, authors feel that it would have been possible to save the life of fetus.

\section{Conclusion:-}

Hereditary Spherocytosis one of the commonest congenital hemolytic anemia may get decompensated during pregnancy and pregnancy complicates the management of Hereditary Spherocytosis. Pregnancy is usually well tolerated and need for splenectomy doesn't arise frequently. Although there is a consensus to subject patient to splenectomy and its inherent risks in clinically moderate and severe HS, the management is still controversial during pregnancy. Given the few studies in literature regarding management of HS during pregnancy, it is hard to formulate guidelines for indications of splenectomy during pregnancy at this juncture. The decision to perform splenectomy in pregnancy is guided at best by the indications in non-pregnant patients of HS. However, authors recommend splenectomy in any pregnant women with HS who is experiencing hemolysis or having symptoms, signs and complications thereof. Second trimester should be preferred for splenectomy. Of note, Obstetricians should exercise high index of suspicion to diagnose this not so uncommon entity as early as pregnancy is diagnosed if a present with signs and symptoms suggestive of hemolysis and plan out further management along with discussing the option of 
splenectomy with patient and her attendants for better perinatal outcome. Not surprisingly, earlier diagnosis and management can commensurate into better outcomes.

\section{References:-}

1. Bolton-Maggs PH, Stevens RF, Dodd NJ, Lamont G, Tittensor P, King MJ. Guidelines for the diagnosis and management of hereditary spherocytosis. Br J Haematol. 2004;126:455-74.

2. Bolton-Maggs PH, Langer JC, Iolascon A, Tittensor P, King MJ. Guidelines for the diagnosis and management of hereditary spherocytosis - 2011 update. Br J Haematol. 2012 Jan;156(1):37-49.

3. Khanna SB, Dash K. Hereditary spherocytosis with pregnancy - a case report. J ObstetGynecol India. 2011 Apr;61(2):205-7.

4. Minkowski O: Übereinehereditäre, unterdemBildeeineschronischenIkterusmitUrobilinurie, Splenomegalie und Nierensiderosisverlaufende Affection. VerhDtschKongr Inn Med. 1900;18:316-9.

5. Chauffard MA. Pathogénie de l'ictèrecongénital de l'adulte. La semainemédicale. 1907;27:25-9.

6. Marchetti M, Quaglini S, Barosi G: Prophylactic splenectomy and cholecystectomy in mild hereditary spherocytosis: analyzing the decision in different clinical scenarios. J Intern Med. 1998;244:217-26.

7. Perrotta S, Gallagher PG, Mohandas N: Hereditary spherocytosis. The Lancet. 2008;372:1411-26.

8. Walensky LD, Narla M, Lux SE: Disorders of the blood cell membrane. In: Handin RI, Lux SE: Principles and practice of hematology. 2nd ed. Philadelphia: Lippincott Williams \& Wilkins, 2003:1753.

9. Bianchi P, Fermo E, Vercellati C,Marcello AP, Porretti L, Cortelezzi A et al. Diagnostic power of laboratory tests for hereditary spherocytosis: a comparison study on 150 patients grouped according to the molecular and clinical characteristics. Haematologica. 2012 Apr;97:516-23.

10. Eber SW, Armbrust R, Schroter W. Variable clinical severity of hereditary spherocytosis: relation to erythrocyticspectrin concentration, osmotic fragility, and autohemolysis. J Pediatr. 1990 Sep;117(3):409-16.

11. Schilling RF: Risks and benefits of splenectomy versus no splenectomy in hereditary spherocytosis - a personal view. Brit J Haematol 2009; 145:723-8.

12. Abdullah F, Zhang Y, Camp M, Rossberg MI, Bathurst MA, Colombani PM, et al. Splenectomy in hereditary spherocytosis: Review of 1,657 patients and application of the pediatric quality indicators. Pediatr Blood Cancer. 2009 Jul;52(7):834-7.

13. Bruni L, Bayas JM, Vilella A, Conesa A. Vaccination coverage in adults undergoing splenectomy: evaluation of hospital vaccination policies. Epidemiol Infect.2006 Aug;134(4):837-44.

14. Pincez T, Guitton C, Gauthier F, de Lambert G, Picard V, Fénéant-Thibault M, et al. Long-term follow-up of subtotal splenectomy for hereditary spherocytosis: a single-center study. Blood. 2016 Mar 24;127(12):1616-8.

15. Moore A, Sherman MM, Strongin MJ. Hereditary spherocytosis with hemolytic crisis during pregnancy. Treatment by splenectomy. Obstet Gynecol. 1976 Jan;47(1):19S-21S.

16. Ho-Yen DO. Hereditary spherocytosis presenting in pregnancy. Acta Heamatol. 1984;72(1):29-33.

17. Maberry MC, Mason RA, Cunningham FG, Pritchard JA. Pregnancy complicated by hereditary spherocytosis. Ostet Gynecol. 1992 May;79(5(Pt1)):735-8.

18. Pajor A, Lehoczky D, Szakacs Z. Pregnancy and hereditary spherocytosis. Report of 8 patients and a review. Arch Gynecol Obstet. 1993;253(1):37-42.

19. Brabec V, Cermak J, Petrtylova K, Jarolim P. Vnitr Lek. 1999 Apr;45(4):220-3.

20. Allran CFJr, Weiss CA3rd, Park AE. Urgent laparoscopic splenectomy in a morbidly obese pregnant woman: case report and literature review. J LaparoendoscAdvSurg Tech A. 2002;12:445-7. 